\title{
Photometric Structure and Star Formation in Blue Compact Dwarf Galaxies
}

\author{
P. Papaderos \\ Institute for Astrophysics, Friedrich-Hund-Platz 1, 37077 Göttingen, Germany
}

The star-formation history and chemodynamical evolution of Blue Compact Dwarf (BCD) galaxies are central issues in dwarf galaxy research. In spite of being old in their vast majority, BCDs resemble in many aspects unevolved low-mass galaxies in the early universe. They are gas-rich (HI mass fraction of typically $>30 \%$ ) and metal-deficient $(7.1 \lesssim 12+\log (\mathrm{O} / \mathrm{H}) \lesssim 8.3)$ extragalactic systems, undergoing intense star-forming $(\mathrm{SF})$ activity within an underlying low-surface brightness (LSB) host galaxy.

A few of the outstanding questions we seek to answer in exploring the photometric structure of BCDs are: i) which intrinsic or environmental properties trigger and control star-forming activity in these mostly isolated systems, ii) how does a BCDs appear in an interburst phase and after its gas reservoir is depleted? Are BCDs active phases in the lifetime of quiescent dwarf irregulars (dIs) and do the latter fade to dwarf ellipticals (dEs) once their gas reservoir has been depleted (see Thuan 1991 and references therein)? iii) what is the role played by the BCD's host galaxy on the SF process? This old stellar component contains, due to its higher mass-to-light ratio, the bulk of the stellar mass in a BCD. Thus, provided that Dark Matter does not dominate within the optical radius of these systems, the LSB host largely determines the gravitational potential within which SF activities occur. It is therefore important to explore its structural and dynamical properties and investigate in which way they may influence the star formation process.

Indeed, studies of the latter question by Papaderos et al. (1996a,b), Noeske et al. (2003) and Papaderos \& Noeske (2006) have revealed significant relations between the structural properties of the LSB and SF component: decomposition of optical surface brightness profiles (SBPs) has shown that SF activities in BCDs do not occur out to a random galactocentric radius but are confined within a characteristic radius which is typically equivalent to two exponential scale lengths of the LSB host. These studies also indicate that the fractional surface of the SF component does not vary randomly but scales inversely with the mass of the LSB host galaxy. This trend suggests a massmorphology relation for BCDs: SF activities within a massive stellar LSB-host are more centrally confined whereas in lower-mass BCDs SF activities are spread over a larger volume of the LSB host.

Such results, if confirmed and extended on a larger BCD sample, will have a strong impact on our understanding of starburst activity in low-mass extragalactic systems in the nearby universe and at high redshift. They suggest that this fundamental process in the cosmos is not entirely stochastic or primarily controlled by the properties of Dark Matter halos but largely regulated through the gravitational potential of the evolving LSB host galaxy.

Noeske, K.G., Papaderos, P., Cairos, L.M., Fricke, K.J. 2003, A\&A 2003, 410, 481 · Papaderos et al. 1996a,b, A\&AS 120, 207; A\&A 314, 59 - Papaderos, P. \& Noeske, K.J. 2006, in prep. . Thuan, T.X. 1991, in Massive Stars in Starbursts, eds. C. Leitherer et al. p. 183.

This work has been supported by Deutsche Forschungsgemeinschaft grant PA 1228/5-1. 